\title{
Regional variation in the flexural properties of the equine hoof wall
}

\author{
AM Goodman ${ }^{1, *}$ and L Haggis ${ }^{2, t}$ \\ ${ }^{1}$ Department of Forensic and Biomedical Sciences, University of Lincoln, Brayford Pool, \\ Lincoln LN6 7TS, UK \\ ${ }^{2}$ Department of Biological Sciences, University of Lincoln, Riseholme Park, Riseholme, \\ Lincoln LN2 2LG, UK \\ *Corresponding author: agoodman@lincoln.ac.uk
}

Submitted 4 November 2008: Accepted 5 April 2009

Research Paper

\begin{abstract}
The equine hoof wall is a hard, keratinous structure that transmits forces generated when the hoof connects the ground to the skeleton of the horse. During locomotion the hoof capsule is known to deform, resulting in an inward curvature of the dorsal wall and expansion of the heels. However, while researchers have studied the tensile and compressive properties, there is a lack of data on the flexural properties of the hoof wall in different locations around the hoof capsule. In this study, the flexural properties and hydration status of the hoof wall were investigated in two orthogonal directions, in different locations around the hoof capsule. The hoof was divided into three regions: the dorsal-most aspect (toe), the medial and lateral regions (quarters) and the heels caudally. Beams were cut both perpendicular (transverse) and parallel (longitudinal) to the orientation of the tubules. Differences in the mechanical properties were then investigated using three-point bending tests. There were considerable differences in the flexural properties around the hoof capsule; transverse beams from the heel were $45 \%$ more compliant than those from the toe region. This corresponded with changes in the hydration of the hoof wall; beams from the heel region were more hydrated $(28.2 \pm 0.60 \%)$ than those from the toe $(24.2 \pm 0.44 \%$; $P<0.01$ ). Regional variation in the water content is thought to help explain differences in the flexural properties. Mechanical data are further discussed in relation to variation in the structure and loading of the hoof wall.
\end{abstract}

Keywords: equine; hoof; stratum medium; mechanical properties; anisotropy

\section{Introduction}

The equine hoof is a hard, keratinous structure that has to be highly resistant to fracture in order to prevent damage to underlying sensitive tissues; it must transmit, dissipate and withstand the forces generated by ground impact without breaking and allow for the process of wear ${ }^{1,2}$. However, this must be achieved without excessive deformation that would damage the sensitive living structures of the hoof ${ }^{3}$. Unlike bone, equine hoof wall has only limited capacity for repair; damaged material can only be replenished by new hoof after it has worn off distally. It is important that the material and structure withstand these forces because lameness in the horse can result from

\footnotetext{
${ }^{\dagger}$ Present address: Writtle College, Lordships Road, Chelmsford,
} Essex, CM1 3RR, UK breakdown in the integrity of the hoof wall material and resulting infection.

During the support phase of stance the hoof capsule deforms, providing a major role in dissipating concussive forces ${ }^{4}$. The hoof wall is an obliquely truncated cone that opens posteriorly between the heels when the hoof contacts the ground and, during the stance phase of locomotion, the dorsal wall of the hoof curves inwards (dorsoconcavity), resulting from longitudinal compressive forces directed parallel to the hoof wall, and the heels expand 5 . The expansion of the heels produces horizontally directed compressive forces in the dorsal surface of the toe and loads the internal wall in tension ${ }^{6,7}$. The dorsal wall of the hoof is effectively flexed inwards, under sufficient loading, and the heels flex outwards during expansion.

The hoof is a complex structure and the material varies in relation to the anatomy of the hoof wall. 
The hoof wall is made up of three layers: the stratum externum, a thin covering thought to inhibit dehydration; the stratum medium, serving a primary mechanical role and the stratum internum, that is responsible for transferring loads to the bony skeleton ${ }^{8}$. The stratum medium, which is the thickest layer, is composed of keratin cells that are organised into two distinct patterns: tubular structures, where the cells' axis lies parallel to the dorsal wall and intertubular material consisting of sheets running parallel to the load-bearing surface of the hoof ${ }^{9}$.

The mechanical properties of biological materials are highly dependent on the water content: a lower water content is associated with a stiffer material ${ }^{10,11}$. The mechanical properties of keratinous materials are also strongly influenced by their hydration status ${ }^{9,12-15}$. Two main hydration gradients have been reported in the equine hoof wall: a horizontal gradient where the outer surface has low levels and the inner, adjacent to the dermis, which maintains higher hydration levels and a vertical gradient where the hydration status increases from the distal surface to the proximal germinative regions ${ }^{9}$. Variation in the hydration status of the hoof wall is thought to be responsible for a stiffness gradient from the inner wall to the dorsal hoof wall ${ }^{9,16}$.

Previous work has established the basic material properties of the hoof wall in compression and tension and some effects of hydration. The present work adds to that foundation by testing the wall in a manner that is close to the deformation in vivo. This study aims to determine whether the flexural properties and water content of the hoof wall vary in different locations around the hoof capsule by testing beams machined from the hoof wall around the whole hoof capsule. We hypothesise that there will be variation in the flexural properties around the hoof wall in different locations around the hoof capsule and that samples taken from the toe region will have a higher yield strength and be composed of a stiffer material than those from the heel region.

\section{Materials and methods}

\section{Specimen preparation}

Five normal equine cadaver lower limbs were collected from the local slaughterhouse (GW Lord and Sons, Gainsborough, UK). Animals were destroyed for reasons other than this study and feet were considered normal where no visible signs of cracks, abscesses, laminitis or gross foot imbalances were apparent. A mixture of three fore- and two hind-limbs were used and the animals were unshod at death. Lower limbs were sealed in plastic bags and placed in a freezer at $-21^{\circ} \mathrm{C}$. After 5 days, hooves were removed from the lower limb and cuts into toe, quarter and heel regions were made using a band saw (Fig. 1a).

Each hoof region was then carefully cut into uniform beams using a band saw and sanded to a uniform thickness using a sanding wheel (Dremel Multi-Model 395, S-B Power Tool Co., New Bern, NC, USA). Beams were $40 \mathrm{~mm}$ in length with a mean width of $3.6 \pm 0.66 \mathrm{~mm}(\mathrm{SD})$ and height of $3.7 \pm 0.66 \mathrm{~mm}(\mathrm{SD})$. In order to investigate mechanical anisotropy of the hoof wall, beams were cut both perpendicular and parallel to the axis of the tubules and termed transverse and longitudinal beams, respectively (Fig. 1b). Material was only taken from the outer stratum medium as defined by Wagner et $a l^{17}$ and beams were cut from the pigmented outer zone the hoof wall consisting of only the first $4 \mathrm{~mm}$ of the material closest to the outer surface of the hoof capsule. This ensured that none of the stratum internum (identified by visual inspection of the position of the laminar junction) was included in the test samples. In order to limit the effects of beam preparation on the in vivo structural arrangement of the outer hoof wall, some transverse samples

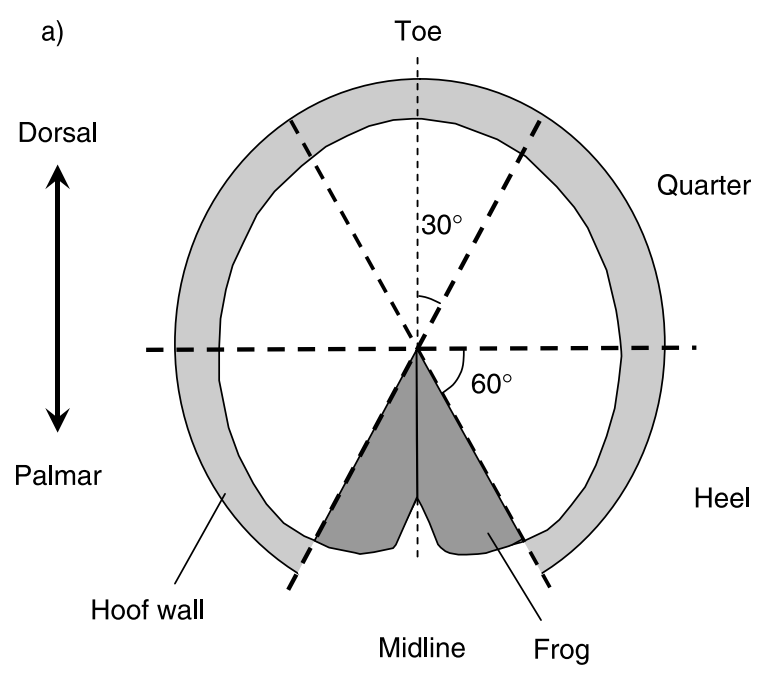

b)

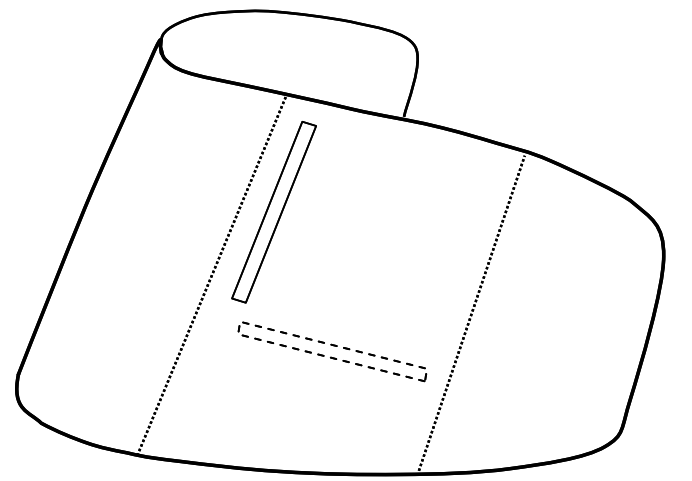

Fig. 1 (a) Distal surface (solar view) of the hoof capsule, indicating hoof regions and (b) the hoof wall to show orientation of the beams relative to the tubules. Transverse beams (---) were cut perpendicular and longitudinal beams $(-)$ were cut parallel to the proximo-distal orientation of the tubules $(\cdots)$ 
exhibited a small degree of natural curvature. It was felt that this would not have a significant effect on the results. Furthermore, to prevent the damaged distal surface of the hoof wall biasing the results, the distal $4 \mathrm{~mm}$ where the hoof contacted the ground, was discarded. Since the palmar aspect of the hoof wall is shorter than the dorsal, it was impossible to cut both longitudinal and transverse beams of sufficient length from the same heel region of each foot. Therefore, transverse and longitudinal samples were taken from opposing regions of the hoof; this was alternated between hooves and data from the medial and lateral samples were pooled. Material from the hoof wall in the toe region was machined into up to four transverse and eight longitudinal beams. To maximise the length of beams, the transverse samples were taken from the most distal region of the hoof wall.

Samples were thawed at room temperature for $24 \mathrm{~h}$, immediately wrapped in damp tissue, placed in sealed, plastic sample bags and stored at $4^{\circ} \mathrm{C}$. The water content of the beams was determined immediately after mechanical testing by measuring mass loss of oven-dried specimens $\left(80^{\circ} \mathrm{C}, 5\right.$ days $)$; water content was calculated per unit fresh weight.

\section{Mechanical tests}

Three-point bending tests (Fig. 2) were performed on each beam cut from the hoof wall using a universal testing machine (Instron, model 4443). Beams were maintained at in vivo moisture content before testing by wrapping in damp tissue and the dimensions were measured at the mid-point using a digital micrometer (Mitutoyo, Japan). The sample was placed between two supports that were spaced at no less than ten times the mid-point depth of the sample to avoid problems with shear ${ }^{18}$. The two supports were $40 \mathrm{~mm}$ apart with the stratum externum facing uppermost. A pushing probe of radius $5 \mathrm{~mm}$

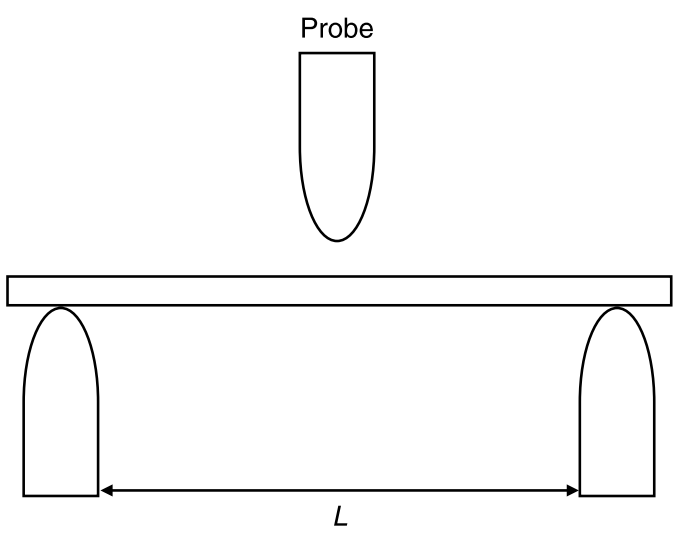

FIG. 2 Three-point bending test. Samples were orientated with the stratum externum uppermost and a pushing probe was lowered causing the sample to bend until it eventually yielded. $L$ is the distance between the supports was lowered at a rate of $30 \mathrm{~mm} \mathrm{~min}^{-1}$ (strain rate of $\sim 6.9 \times 10^{-3} \mathrm{~s}^{-1}$ ), causing the sample to bend until it eventually yielded. This was within the range of physiological strain rates described by Kasapi and Gosline $^{19}$. For longitudinal beams, the force acted perpendicular to the tubular axis in the circumferential plane, and for transverse beams the force acted parallel to the tubular axis in the radial plane.

An interfaced computer was used to produce a graph of applied force versus displacement, permitting calculation of the mechanical properties of the sample.

\section{Analysis of bending tests}

The bending yield strength, $S$ [equation (1)] and bending modulus $E$ [equation (2)], of the material of which they were composed, were calculated using well-known equations ${ }^{20}$. The yield strength in bending, $S$, is given by the following expression:

$$
\text { Yield strength bending }=3 F_{\text {yield }} L / 2 b b^{2}
$$

where $F_{\text {yield }}$ is an estimate of the point at which the hoof wall starts to yield, $L$ is the distance between the supports and $b$ and $b$ are the width and height, respectively, of the beam's cross-section in metres. The yield point was estimated using a stiffness reduction technique; the initial linear slope of the original force versus displacement curve was reduced by $10 \%$, plotted and the intersection with the force versus displacement curve defined the yield point ${ }^{17}$ (Fig. 3).

The bending modulus, $E$, is given by

$$
\text { Bending modulus }=L^{3}(\mathrm{~d} F / \mathrm{d} \delta) / 4 b b^{3} \text {, }
$$

where $\mathrm{d} F / \mathrm{d} \delta$ is the initial slope of the force versus displacement curve. A high modulus indicates a stiffer material.

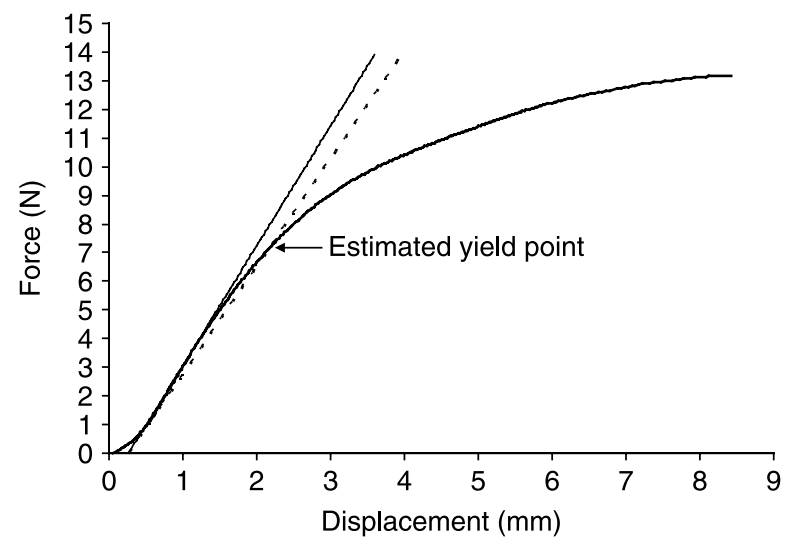

FIG. 3 Typical force versus displacement curve for equine hoof wall indicating yield point estimation technique. The initial linear slope of the original force versus displacement curve was reduced by $10 \%$ and plotted (---). The intersection with the force versus displacement curve defined the yield point 


\section{Statistical analysis}

Analysis of variance was used to investigate variation in the water content and mechanical properties of the hoof wall. The water content data were normalised using an arcsine square root transformation before analysis. Significant $(P<0.05)$ changes in the mechanical properties and transformed water content data between the regions were further investigated by using a Tukey-Kramer multiple comparison test ${ }^{21}$. In order to investigate anisotropic behaviour of the hoof wall, paired Student's $t$-tests were used to test for significant differences in the bending modulus and yield strength of longitudinally and transversely orientated beams. Regression analysis was used to investigate the relationship between the bending modulus and the water content of the beams. Unless otherwise stated, all values in the text are means $\pm \mathrm{SE}$.

\section{Results}

\section{Mechanical bebaviour}

A typical force versus displacement plot for the hoof wall is shown in Fig. 3; hoof wall samples produced a characteristic biphasic force versus displacement curve. There was an initial linear elastic relationship between force and displacement followed by a plateau as the material started to yield. The bending modulus (mean \pm SD) of the hoof wall material in transverse beams was: toe $380 \pm 61.6$ $\mathrm{MPa}$; quarters $309 \pm 42.2 \mathrm{MPa}$ and heel $210 \pm 52.2 \mathrm{MPa}$; and in longitudinal beams: toe $338 \pm 26.2 \mathrm{MPa}$; quarters $361 \pm 52.8 \mathrm{MPa}$ and heel $265 \pm 37.3 \mathrm{MPa}$. The bending yield strength (mean \pm SD) of transverse beams was: toe $10.8 \pm 1.81 \mathrm{MPa} ;$ quarters $10.1 \pm 2.63 \mathrm{MPa}$ and heel $7.8 \pm 0.96 \mathrm{MPa}$; and of longitudinal beams: toe $10.0 \pm 0.48 \mathrm{MPa} ;$ quarters $10.8 \pm 1.36 \mathrm{MPa}$ and heel $7.9 \pm 1.07 \mathrm{MPa}$. The water content (mean $\pm \mathrm{SD}$ ) of transverse beams was: toe $24.2 \pm 0.98 \%$; quarters $25.1 \pm 0.56 \%$ and heel $28.2 \pm 1.34 \%$; and of longitudinal beams was: toe $30.5 \pm 4.27 \%$; quarters $27.5 \pm 1.67 \%$ and heel $29.9 \pm 1.82 \%$.

\section{Site variation around the boof capsule}

There were significant differences in the mechanical properties of the wall material around the hoof capsule; beams taken from heels were significantly more compliant and weaker than those taken from the toe and quarter regions (Fig. $4 \mathrm{a}$ and $4 \mathrm{~b}$; Table 1). However, there were subtle, but significant regional differences in the properties of beams between those cut perpendicular and parallel to the tubular axis. Transverse beams cut from the heels of the hoof wall were more compliant, around $45 \%$, but not significantly weaker $(P=0.076$; $n=5$ ) than those cut from the toe and quarter regions, whereas longitudinal beams in the heel region were around $22 \%$ more compliant and $21 \%$ weaker than those from the toe $(P<0.01$; Table 1 ; Fig. $4 \mathrm{a}$ and $4 \mathrm{~b})$.
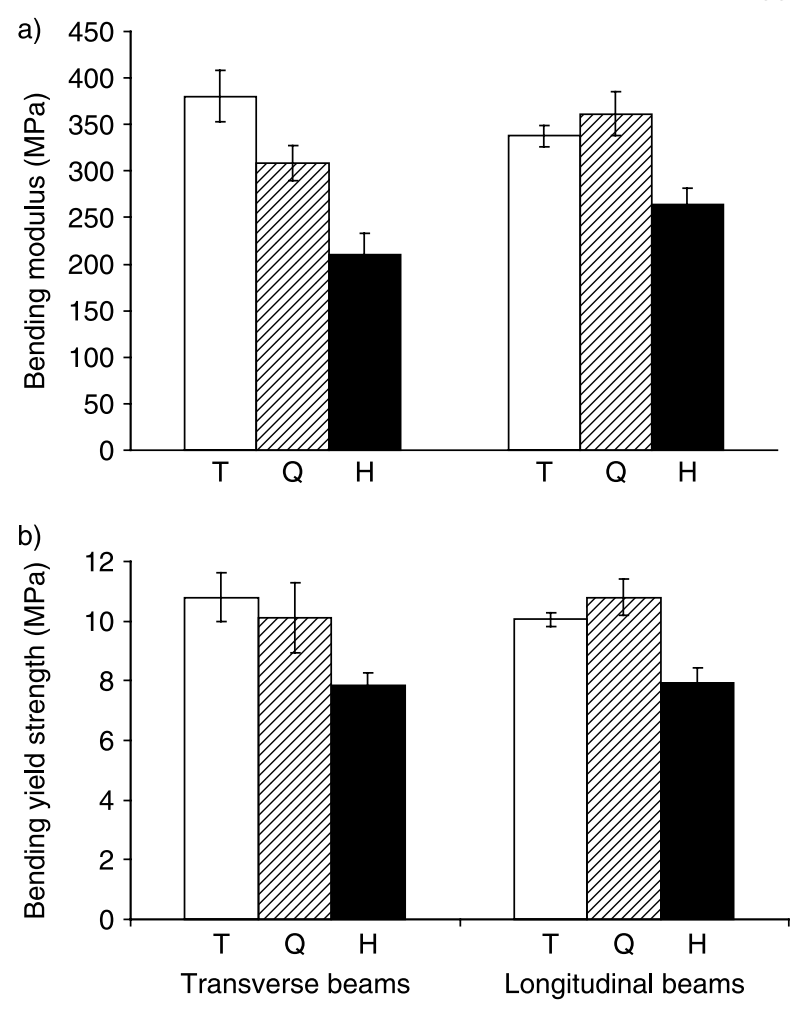

FIG. 4 The mechanical properties of the hoof wall, in two orthogonal directions, in different locations around the hoof capsule: the toe $(T)$, quarters $(Q)$ and heel $(H)$ regions. There were significant differences in (a) the bending modulus and (b) the bending yield strength of the wall material around the hoof capsule; beams taken from heels were significantly more compliant than those cut from the toe and quarter regions, but only longitudinal beams were significantly weaker $(P<0.01)$. The results were analysed using ANOVA, $n=5$. Vertical bars indicate \pm SE

Differences in properties corresponded with significant differences in the water content of the hoof wall between regions; transverse beams taken from the heels were significantly more hydrated than those from the toe and quarters (Fig. 5; $P<0.01$ ). The water content of transverse beams from the heel region was

Table 1 Results of Tukey-Kramer multiple comparison tests showing regional differences in the properties of the hoof wall between the toe $(T)$, quarters $(Q)$ and heels $(H)$, and paired Student's $t$-tests showing differences between transverse (Tr) and longitudinal $(\mathrm{L})$ beams

\begin{tabular}{|c|c|c|c|}
\hline \multicolumn{4}{|c|}{ Tukey-Kramer multiple comparison tests } \\
\hline Properties/orientation & Transverse & Longitudinal & \\
\hline $\begin{array}{l}\text { Bending modulus (MPa) } \\
\text { Bending yield strength (MPa) } \\
\text { Water content (\%) }\end{array}$ & $\begin{array}{l}\mathrm{H}<\mathrm{T} \mathrm{Q}^{\mathrm{a}} \\
\mathrm{NS} \\
\mathrm{H}>\mathrm{T} \mathrm{Q}^{\mathrm{a}}\end{array}$ & $\begin{array}{l}\mathrm{H}<\mathrm{TQ}^{\mathrm{a}} \\
\mathrm{H}<\mathrm{TQ}^{\mathrm{a}} \\
\mathrm{NS}\end{array}$ & \\
\hline \multicolumn{4}{|c|}{ Paired Student's $t$-tests } \\
\hline Properties/region & Toe & Quarters & Heel \\
\hline $\begin{array}{l}\text { Bending modulus (MPa) } \\
\text { Bending yield strength (MPa) } \\
\text { Water content (\%) }\end{array}$ & $\begin{array}{l}\mathrm{NS} \\
\mathrm{NS} \\
\mathrm{L}>\mathrm{Tr}^{\mathrm{b}}\end{array}$ & $\begin{array}{l}L>\operatorname{Tr}^{b} \\
\text { NS } \\
\text { NS }\end{array}$ & $\begin{array}{l}\text { NS } \\
\text { NS } \\
\text { NS }\end{array}$ \\
\hline
\end{tabular}

NS, non-significant; $n=5 ;{ }^{\mathrm{a}} P<0.01 ;{ }^{\mathrm{b}} P<0.05$. 
c. $17 \%$ more hydrated than that of the toe. However, in contrast to transverse beams, longitudinal beams showed no significant differences in their water content between the regions (Fig. 5; $P>0.05$ ).

\section{Beam orientation and anisotropic bebaviour}

There were significant differences between the stiffness and the water content of beams cut parallel and perpendicular to the tubular axis. Paired Student's $t$-tests showed that the hoof wall was anisotropic; longitudinal beams in the quarters were $17 \%$ stiffer than transverse beams (Table 1 ; Fig. $4 \mathrm{a} ; P<0.05$ ). However, there were no significant differences between the stiffness of longitudinal and transverse beams in the toe or heels (Table 1; Fig. 4a; $P>0.05$ ). There were also significant differences in the water content between beams cut parallel and those cut perpendicular to the tubular axis; longitudinal beams from the toe region were $26 \%$ more hydrated than transverse beams taken from the same region (Fig. 5; Table $1 ; P<0.05$ ).

\section{Relationship between flexural properties and water content}

Regression analysis showed that the bending modulus decreased significantly with an increase in water content $\left(t_{13}=-5.38, P<0.001, r^{2}=69.0 \%\right)$; transverse beams with a higher water content were more compliant (Fig. 6a). The line of best fit is given by the equation:

Bending modulus $(\mathrm{MPa})=1227-[35.9$

$$
\times \text { water content }(\%)] \text {. }
$$

However, for longitudinal beams, there was no significant relationship between the water content and the bending modulus (Fig. 6b, $t_{13}=-0.55, P>0.05$, $\left.r^{2}=2.3 \%\right)$.

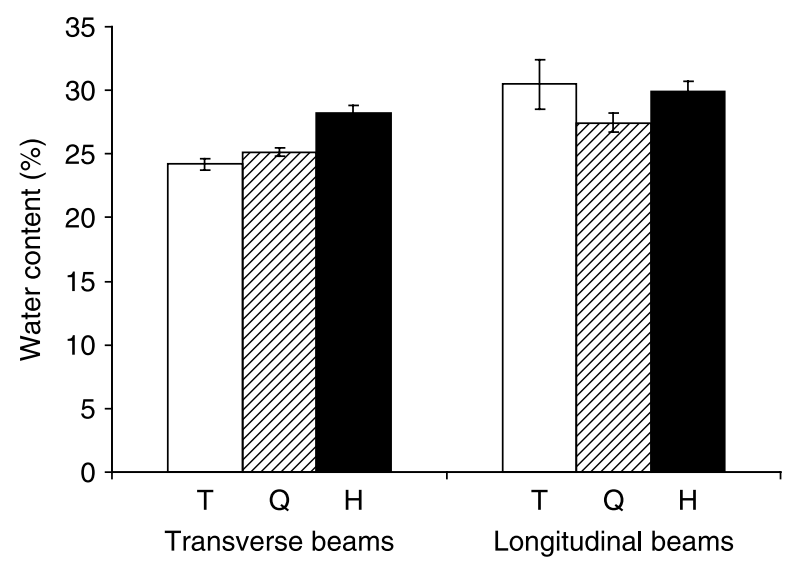

FIG. 5 Variation in the water content of transverse and longitudinal beams taken from the hoof wall around the capsule: the toe $(T)$, quarters $(Q)$ and heel $(H)$ regions. Transverse beams taken from the heels were significantly more hydrated than those from the toe and quarters $(P<0.01)$. The results were analysed using ANOVA, $n=5$. Vertical bars indicate \pm SE

\section{Discussion}

This study shows that there is significant regional variation in the flexural properties of the hoof wall; beams taken from the heel were significantly more compliant than those taken from the toe and the quarter regions. This is important because previous studies have shown that when the hoof contacts the ground during locomotion, the hoof capsule deforms during the support phase of stance to dissipate concussive forces. During the stance phase of locomotion, the dorsal wall of the hoof curves inwards (dorsoconcavity), resulting from longitudinal compressive forces directed parallel to the hoof wall, and the heels expand $^{5}$. It is not surprising that beams taken from the heel region were more flexible, as this would facilitate the outward flexing of the heels during the expansion of the hoof capsule. This is supported by previous work where samples from the medial and lateral regions were more compliant, in both tension and compression, than those from the dorsal region ${ }^{22}$. Experiments using photoelastic stress analysis to investigate the relative position of strain field epicentres around the hoof capsule also showed that the majority of strain field epicentres were evenly
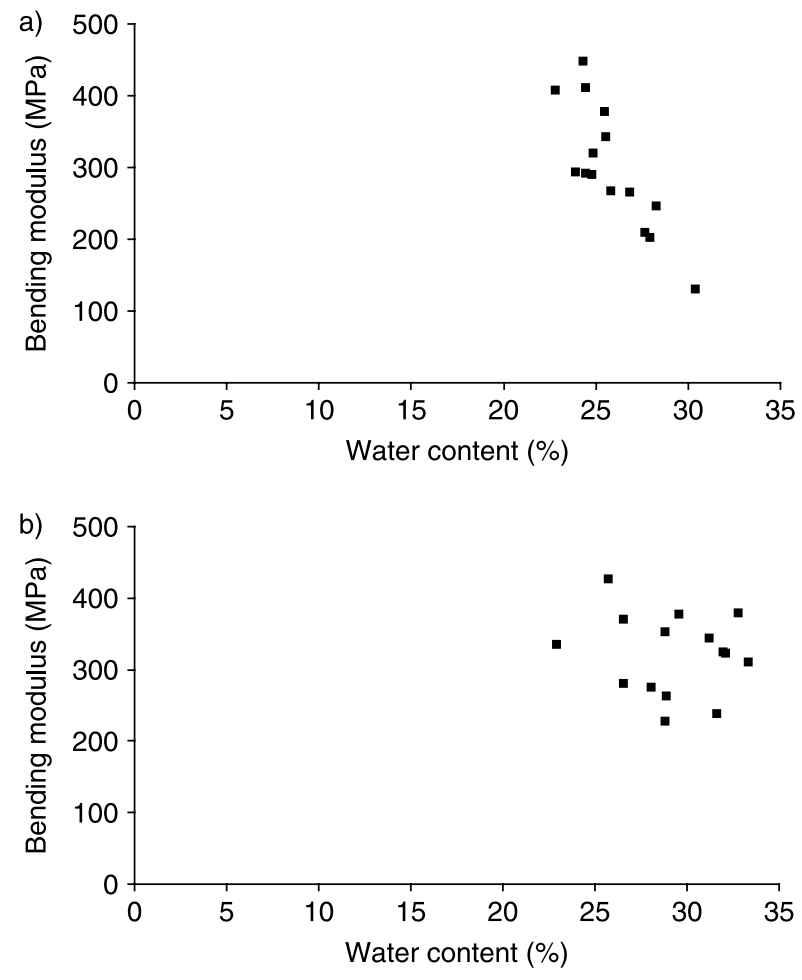

FIG. 6 Relationship between the water content and bending modulus of (a) transverse and (b) longitudinal samples taken from the hoof wall. There was a significant negative association between the water content and the stiffness of transverse samples; the bending modulus decreased significantly with an increase in water content $\left(t_{13}=-5.38, P<0.001, r^{2}=69.0 \%\right)$ 
distributed on both sides of the hoof from the heel to the junction of the quarters and toe region; relatively few epicentres were recorded in the toe region ${ }^{23}$.

Variation in the mechanical properties around the hoof capsule and anisotropy might be explained by two possible factors: first, the structure of the hoof wall or, secondly, variation in the chemical nature of keratin. First, differences in the morphology and ultrastructure of the wall material are likely to affect its mechanical properties around the hoof capsule. The hoof wall is made of keratin, a fibre-reinforced composite material composed of $\alpha$-keratin intermediate filaments embedded in a viscoelastic protein matrix, and the intermediate filaments are composed of protofibrils that associate helically in super coils $^{12,24}$. This in turn is organised into cylindrical structures, called tubules, which are orientated longitudinally, parallel to the hoof wall. It may be that variation in the ultrastructure - i.e. orientation of the intertubular intermediate filaments around the hoof capsule - is responsible, at least in part, for the circumferential variation in mechanical properties and anisotropic behaviour. In this study, there was evidence of mechanical anisotropy with significant differences between the mechanical properties of samples relative to the orientation of the tubules. Longitudinal beams from the quarters were $17 \%$ stiffer than transverse beams (Fig. 4a; Table 1; $P<0.05$ ). This is likely to result from the structural organisation of the hoof wall material. Previous work on the ultrastructure and mechanical properties of the hoof wall in the toe region showed that the orientation of the intertubular intermediate filaments varied between the layers of the hoof wall; the plane of intermediate filaments changes from perpendicular to the tubule axis, in the inner layer, to parallel in the outer layer ${ }^{25}$. This prevented the propagation of cracks and would also be an important factor in anisotropic behaviour of the hoof wall.

The significance of tubule density on the mechanical properties of the equine hoof has previously been suggested by Leach and Zoerb $^{3}$ and investigated by Kasapi and Gosline ${ }^{26}$. A radial increase in tubule density from the inner to the outer surface of the hoof wall has been shown to correspond to the increasing stiffness gradient observed from the inner to outer surface of the hoof wall ${ }^{25,27}$. Furthermore, tubule morphology and orientation has been shown to be related to the direction of loading; tubules in the outer layer of the equine hoof wall are elliptical in cross-section and the main axis is oriented circumferentially, whereas those in the inner layers are circular in cross-section. The elliptical tubules would appear to be optimised to minimise the effects of lateral compressive stress ${ }^{3}$.

Secondly, it is possible that circumferential variation in physical properties is determined at the molecular level. In hard keratins, cysteine-cysteine disulphide cross-links stabilise the microfibrils and cysteine-rich proteins of the matrix ${ }^{12}$. The rest of the matrix has few covalent cross-links and its stabilisation is dependent on weaker hydrogen bonds that are hydration dependent $^{9}$. Just as an increase in the quantity of non-cysteine protein may decrease keratin stiffness (in the presence of water), so an increase in cysteine residues and the resultant disulphide bonds with which they are associated could increase flexural stiffness and strength. A greater proportion of the stronger disulphide bonds within the protein matrix that cements the hoof together could increase both the stiffness and the strength of the hoof capsule. Thus, regional variation in the composition of the protein matrix that cements the keratin fibres of the hoof together could be an important factor in determining its mechanical properties.

Not surprisingly, variation in the flexural properties around the hoof capsule corresponded with variation in the water content; transverse samples from the heel region, which were more compliant, were also more hydrated than those from the toe region. Regression analysis of the relationship between the water content and the stiffness supports this; in transverse samples, there was a significant negative association between the water content and the stiffness of the samples (Fig. 6a). Similarly, longitudinal beams cut from the hoof wall in the heel region were also more compliant and weaker than those from the toe; however, variation in the stiffness of the longitudinal beams could not be accounted for by variation in water content; there was no significant relationship between the water content and the stiffness of the material (Fig. 6b). This may be due to increased variation in the properties of longitudinal samples resulting from a proximo-distal water gradient ${ }^{2}$. In addition, longitudinal beams from the toe region were significantly more hydrated than transverse beams taken from the same region. It is possible that this was a result of the increased number of exposed tubule cross-sections in the transverse samples ${ }^{26}$ or the combined effects of the sampling regime and presence of a proximo-distal water gradient in the hoof wall ${ }^{2}$. However, the water content of hoof wall samples in this study of $27.5 \pm 0.3 \%$ was similar to values recorded by others: Butler and $\mathrm{Hintz}^{28}$ of $27.8 \%$ and Douglas et $a l^{22}$ of $27.9 \%$. Furthermore, hoof wall material showed typical force versus displacement behaviour previously described in the equine hoof ${ }^{1,29}$.

Whatever the causal mechanisms for the regional differences found in this study, it may be concluded that the results support the concept that the material properties are linked to known patterns of deformation in the hoof wall. The presence of an increasing 
gradient of stiffness circumferentially from the heels to the toe region may perform a damping function, smoothing the transfer of energy between areas of high and low deformation (strain). As the foot usually comes to the ground heel first, the significant and rapid expansion of the heels would be resisted by the progressively stiffer material of the quarter and toe regions.

Furthermore, if one considers the break-over of the foot where the dorsal wall is subjected to the resistance of the ground surface, excessive longitudinal flexion of the dorsal hoof wall not only reduces the efficiency of locomotion but could result in excessive forces concentrated in the distal portion of the laminar junction. A stiffer dorsal hoof wall would help prevent such a localised stress concentration and hence reduce the risk of peeling the hoof wall from the phalanx, much like bending back a fingernail.

This study supports the hypothesis that there is significant circumferential variation in the mechanical properties and water content of the hoof wall. Patterns of variation are in accord with previous work on the deformations experienced by each region of the wall under load. It is important that future work determines whether the ultrastructure and biochemical composition of the hoof wall also varies around the hoof capsule.

\section{Acknowledgements}

We thank Gary Millar and Amanda Mylett for technical support and the referees for their constructive comments.

\section{References}

1 Zoerb GC and Leach DH (1978). Mechanical properties of the hoof wall of the horse. American Society of Agricultural Engineers (ASAE) technical paper, No. 78-3063 pp. 1-14.

2 Bertram JEA and Gosline JM (1986). Fracture toughness design in horse hoof keratin. Journal of Experimental Biology 125: 29-47.

3 Leach DH and Zoerb GC (1983). Mechanical properties of equine hoof wall tissue. American Journal of Veterinary Research 44: 2190-2194.

4 Dyhre-Poulsen P, Smedegaard HH, Roed J and Korsgaard E (1994). Equine hoof function investigated by pressure transducers inside the hoof and accelerometers mounted on the first phalanx. Equine Veterinary Journal 26: 362-366.

5 Lungwitz A (1891). The changes in the form of the horse's hoof under the action of bodyweight. Journal of Comparative Pathology and Therapeutics 4: 191-211.

6 Thomason JJ, Biewener AA and Bertram JEA (1992). Surface strain on the equine hoof wall in vivo: implication for the material design and functional morphology of the wall. Journal of Experimental Biology 166: 145-168.
7 Newlyn HA, Collins SN, Cope BC, Hopegood L, Latham RJ and Reilly JD (1998). Finite element analysis of static loading in donkey hoof wall. Equine Veterinary Journal Supplement 26: 103-110.

8 Stump JE (1967). Anatomy of the normal equine foot, including microscopic features of the laminar region. Journal of the American Veterinary Medical Association 151: 1588-1598.

9 Bertram JEA and Gosline JM (1987). Functional design of horse hoof keratin: the modulation of mechanical properties through hydration effects. Journal of Experimental Biology 130: 121-136.

10 Kitchener A and Vincent JFV (1987). Composite theory and the effect of water on the stiffness of horn keratin. Journal of Material Science 22: 1385-1389.

11 Niklas KJ (1992). Voigt and Reuss models for predicting changes in Young's modulus of dehydrating plant organs. Annals of Botany 70: 347-355.

12 Fraser RDB and Macrae TP (1980). Molecular structure and mechanical properties of keratins. In: Vincent JFV and Currey JD (eds) The Mechanical Properties of Biological Materials. Symposium of the Society for Experimental Biology, XXXXIV, Cambridge, UK: Cambridge University Press, pp. 211-246.

13 Collins SN, Cope BC, Hopegood L, Latham RJ, Linford RG and Reilly JD (1998). Stiffness as a function of moisture content in natural materials: characterisation of hoof horn samples. Journal of Materials Science 33: 5185-5191.

14 Bonser RHC and Farrent JW (2001). Influence of hydration on the mechanical performance of duck down feathers. British Poultry Science 42: 271-273.

15 Hinterhofer C, Stanek C and Binder K (1998). Elastic modulus of equine hoof horn, tested in wall samples, sole samples and frog samples at varying levels of moisture. Berliner und Münchener Tierärztliche Wochenschrift 111: 217-221.

16 Wagner IP and Hood DM (2002). Effect of prolonged water immersion on equine hoof epidermis in vitro. American Journal of Veterinary Research 63: 1140-1144.

17 Wagner IP, Hood DM and Hogan HA (2001). Comparison of bending modulus and yield strength between outer stratum medium and stratum medium zona alba in equine hooves. American Journal of Veterinary Research 62: 745-751.

18 Vincent JFV (1992). Biomechanics-materials: A Practical Approach. Oxford, UK: IRL Press at Oxford University Press, pp. 247.

19 Kasapi MA and Gosline JM (1996). Strain-rate-dependent mechanical properties of the equine hoof wall. Journal of Experimental Biology 199: 1133-1146.

20 Jackson AP (1992). Bone, nacre and other ceramics. In: Vincent JFV (ed.) Biomechanics-materials: A Practical Approach. Oxford, UK: IRL Press at Oxford University Press, pp. 33-56.

21 Sokal RR and Rohlf FJ (1995). Biometry: The Principles and Practice of Statistics in Biological Research. 3rd edn. New York: W.H. Freeman and Company, pp. 887.

22 Douglas JE, Mittal C, Thomason JJ and Jofriet JC (1996). The modulus of elasticity of equine hoof wall: implications for the mechanical function of the hoof. Journal of Experimental Biology 199: 1829-1836.

23 Dejardin LM, Arnoczky SP and Cloud GL (1999). A method for determination of equine hoof strain patterns using photoelasticity: an in vitro study. Equine Veterinary Journal 31: 232-237.

24 Crewther WG, Dowling LM, Steinert PM and Parry DAD (1983). Structure of intermediate filaments. International Journal of Biological Macromolecules 5: 267-274.

25 Kasapi MA and Gosline JM (1997). Design complexity and fracture control in the equine hoof wall. Journal of Experimental Biology 200: 1639-1659. 
26 Kasapi MA and Gosline JM (1998). Exploring the possible functions of equine hoof wall tubules. Equine Veterinary Journal Supplement 26: 10-14.

27 Reilly JD, Collins SN, Cope BC, Hopegood L and Latham RJ (1998). Tubule density of the stratum medium of horse hoof. Equine Veterinary Journal Supplement 26: 4-9.
28

Butler KD and Hintz HF (1977). Effect of level of feed intake and gelatin supplementation on growth and quality of hoofs of ponies. Journal of Animal Science 44: 257-261.

29 Kasapi MA and Gosline JM (1999). Micromechanics of the equine hoof wall: optimizing crack control and material stiffness through modulation of the properties of keratin. Journal of Experimental Biology 202: 377-391. 